\title{
Mechanofluorochromic properties of an AIEE-Active 2-Phenylbenzoxazole Derivative: More than Meets the Eye?
}

\author{
Chantal Carayon, ${ }^{[a]}$ Abdelhamid Ghodbane,${ }^{[a]}$ Nadine Leygue, ${ }^{[a]}$ Jinhui Wang, ${ }^{[a, b]}$ Nathalie Saffon- \\ Merceron, ${ }^{[\mathrm{c}]}$ Ross Brown, ${ }^{[\mathrm{d}]}$ Suzanne Fery-Forgues* ${ }^{[\mathrm{a}]}$ \\ [a] SPCMIB, UMR 5068, CNRS-Université de Toulouse III Paul Sabatier, 118 route de Narbonne, \\ Toulouse, 31062 (France).E-mail: sff@chimie.ups-tlse.fr \\ [b] Institute of Drug Discovery Technology, Ningbo University, Ningbo 315211 (China) \\ [c] Service commun RX, Institut de Chimie de Toulouse, ICT-FR2599, Université de Toulouse III Paul \\ Sabatier, 31062 Toulouse cedex 9 (France) \\ [d] CNRS/Univ Pau \& Pays Adour/ E2S UPPA, Institut des Sciences Analytiques et de Physicochimie pour \\ l'Environnement et les Matériaux, UMR 5254, 64000 Pau (France)
}

\author{
Keywords: \\ aggregation-induced emission \\ benzoxazole \\ fluorescence \\ mechanofluorochromism \\ waveguiding
}

Stack it up: Addition of a dicyanovinyl group onto a 2-phenylbenzoxazole framework led to the emergence of aggregation-induced emission enhancement and phase-transition mechanofluorochromic properties. However, the spectroscopic effects also depend on the size, compactness and heterogeneity of the sample.

\section{ABSTRACT}

The presence of a dicyanovinyl group on the robust 2-phenylbenzoxazole framework led to the emergence of aggregation-induced emission enhancement (AIEE) characteristics. A mechanofluorochromic (MFC) behaviour was observed. The pristine microcrystals emitted yellowgreen light, which became golden yellow upon grinding, with the reverse process observed upon heating or solvent fuming. This phenomenon was accompanied by a reversible evolution of the photoluminescence quantum yields and lifetimes. These variations were attributed to the formation of different types of emissive species in the crystalline phases and in the ground material, which is a mixture of very small microcrystals and amorphous matter. Remarkably, evidence was also given for other spectroscopic effects, which depend on the size, compactness and heterogeneity of the solid samples. These effects are presumably linked to the generation of regions rich in low-energy exciton traps in polycrystalline and pressed samples, and to light reabsorption. They are enhanced by the fact that the pristine platelets exhibit self-waveguided edge emission, and they are all the more significant as the MFC effect is rather weak.

\section{Introduction}

Stimuli-responsive organic materials that exhibit fluorescence emission changes upon mechanical stress, i.e. mechanofluorochromic (MFC) materials, have recently gained increasing attention both in the fundamental research field of solid photophysics and in the applied field of 
polymer stress sensors, security inks, data storage and memory devices. ${ }^{[1-7]}$ It is now widely acknowledged that the reversible emission colour transformation results from changes of solid-state molecular interactions: the molecular packing is affected by grinding, pressing or shearing, and then restored to its original state by annealing or solvent fuming, in the absence of any chemical reaction. ${ }^{\left[{ }^{8-}\right.}$ ${ }^{10]}$ A prerequisite is therefore a loose molecular stacking in the crystal state, which can be easily destroyed under mechanical stimuli. Among the many MFC molecules reported to date, those containing non-planar $\pi$-conjugated moieties, or aromatic groups linked by a single bond, are good candidates, especially when appended chemical groups increase the number of possible conformations in the solid state. ${ }^{[1-26]}$ Such molecules are often derivatives of aggregation-induced emission (AIE) fluorogens. ${ }^{[27]}$

The aim of the present work is to study mechanofluorochromism in the 2-phenylbenzoxazole (PBO) series. Due to their excellent chemical, thermal and photochemical stability, and unique emission properties, $\mathrm{PBO}$ derivatives are widely used as fluorescent dyes in solution and in polymers, and their value for solid-state fluorescence is now well established. ${ }^{[28]}$ Many of them become AIE fluorogens after enlargement of their $\pi$-conjugated system by a $-\mathrm{CH}=\mathrm{CR}^{1} \mathrm{R}^{2},-\mathrm{CH}=\mathrm{NR}$ or $-\mathrm{N}=\mathrm{CR}^{1} \mathrm{R}^{2}$ group, or after insertion of a $-\mathrm{NH}-\mathrm{C}(\mathrm{O})-\mathrm{R}$ group on the phenyl ring, $\mathrm{R}$ and $\mathrm{R}^{1}$ being an unsaturated or aromatic moiety. However, to the best of our knowledge, bis(benzoxazolic)stilbene is the only PBO derivative for which MFC has been reported. ${ }^{[29-31]}$ Within the wider family of benzoxazole derivatives, a few examples can be found among molecules that contain an aromatic moiety with bowl-shape configuration $^{[14-16]}$ or a difluoroboron $\beta$-iminoenolate group. ${ }^{[32,33]}$ We show here that the presence of a dicyanovinyl group on the phenyl ring is enough to promote the appearance of such a behaviour in a quasi-planar PBO derivative (Compound 1, Figure1). Owing to the careful observation of the sample morphology, this compound has also proved to be very useful to study what could possibly be hidden behind conventional MFC effect.

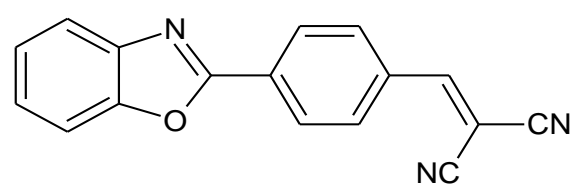

Figure. 1. Chemical structure of compound $\mathbf{1 .}$

\section{Results and Discussion}

\subsection{Crystallographic Study}

Compound 1 was obtained in good yields by reacting the aldehyde of PBO with malononitrile. The slow evaporation of an ethanol solution led to the formation of pale yellow, diamond-shaped crystals, suitable for X-ray analysis. Compound $\mathbf{1}$ crystallized in the triclinic $P \overline{1}$ system. The crystal unit cell contained two molecules. The PBO framework was planar, as already noticed for various derivatives of this compound ${ }^{[34-38]}$ and closely-related 2-phenylnaphthoxazole ${ }^{[39]}$ derivatives, and the dicyanovinyl group formed a small dihedral angle of around $11^{\circ}$ with the rest of the molecule. Each molecule shared short contacts with six neighbours located on the same plane. These strong $\mathrm{N} \cdots \mathrm{H}-\mathrm{C}$ hydrogen bonds (blue dotted lines) were formed between the cyano groups and the hydrogen atoms of 
the vinylene unit and benzozaxole ring. As a result, all molecules pointed in the same direction and formed layers (Figure 2a). Each molecule also formed an antiparallel dimer with a molecule situated in the overlying layer (Figure 2b), $3.4 \AA$ away. Remarkably, the molecules were shifted laterally, so that their aromatic systems did not fully overlap (Figure 2c).

a

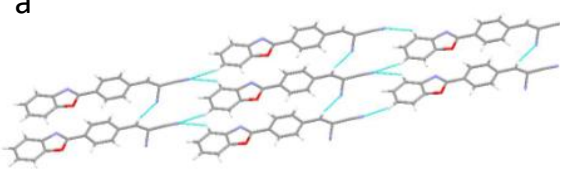

b
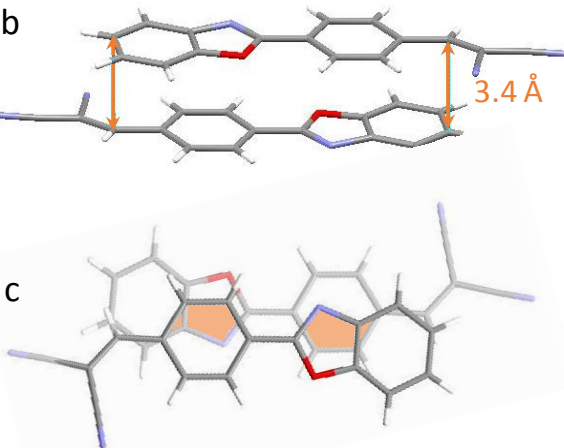

Figure 2. Crystal packing mode of compound 1. Molecules displayed in the same layer (a), antiparallel dimer (b) and face-on view (c) of stacked molecules. Short contacts are in turquoise ink, the pink colour indicates the overlap of the aromatic systems.

\subsection{Spectroscopy in Solution}

A rapid overview of the spectroscopic behaviour of compound $\mathbf{1}$ in solution is given by the study of this compound in three solvents of increasing polarity and proticity, i. e. n-heptane, tetrahydrofuran (THF) and ethanol (Table S1, Supporting Information). The absorption spectra were situated in the near-UV. Fine vibronic structure was only observed in $n$-heptane (Figure 3). The molar extinction coefficient $\varepsilon$ was measured to be $37600 \mathrm{M}^{-1} \mathrm{~cm}^{-1}$ in ethanol at the maximum absorption wavelength.

Compound 1 weakly emitted violet to turquoise light depending on the solvent. The emission spectra were the mirror images of the absorption spectra, showing fine vibrational resolution in $n$ heptane and a broad unresolved band in THF and ethanol. The maxima shifted from $419 \mathrm{~nm}$ to $490 \mathrm{~nm}$ with increasing polarity, resulting in a large Stokes shift in THF and ethanol. Most likely, this strong positive solvatochromism results from the presence of the electron-withdrawing dicyanovinyl group, which enhances charge transfer in the excited state. But, the detrimental effect of this group is that the fluorescence quantum yield was very low (0.02-0.03) in the three solvents, thirty times lower than for the alkyl derivatives of PBO. ${ }^{[34]}$

Increasing from 0 to $90 \%$ the proportion of glycerol in an ethanol solution of $\mathbf{1}$ led to a strong enhancement of the emission efficiency (Figure 3b). This viscosity effect suggests that $\mathbf{1}$ behaves like a molecular rotor, as reported for a long time for many compounds bearing a dicyanovinyl group, which are used as molecular viscosity probes for various applications. ${ }^{[40-43]}$ It is well established that upon optical excitation these molecules initially form an intramolecular charge-transfer (ICT) state 
with partial electron transfer, then they undergo a twist rotation around the single bond accompanied by charge separation. The resulting twisted intra-molecular charge-transfer (TICT) state may promote non-radiative deactivation, which strongly competes with fluorescence emission. It has been recently shown that rotation may also occur around the double bond, also leading to efficient deactivation, depending on the compound and environment polarity. ${ }^{[11,42]}$
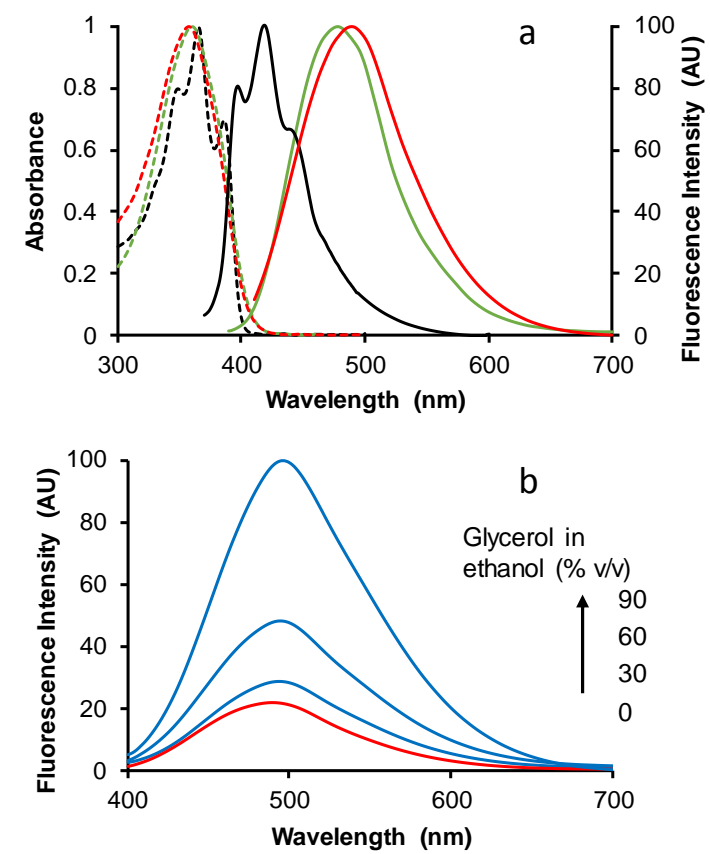

Figure 3. a) Normalized absorption (dashed lines) and emission (solid lines) spectra of compound 1 in $n$-heptane (black lines), THF (green lines) and ethanol (red lines). Solution concentration about $2.6 \times 10^{-5} \mathrm{M}$ for absorption. For emission, $\lambda_{\mathrm{ex}}=348 \mathrm{~nm}$ in $\mathrm{n}$-heptane and $380 \mathrm{~nm}$ in THF and ethanol. b) From bottom to top: Emission spectra in ethanol and in 30, 60 and $90 \% \mathrm{v} / \mathrm{v}$ glycerol/ethanol mixtures, $\lambda_{\mathrm{ex}}=380 \mathrm{~nm}$.

\subsection{Adsorption on a Nylon Filter}

Nylon filters dipped in an ethanol solution of $\mathbf{1}\left(2.6 \times 10^{-5} \mathrm{M}\right)$ and left to dry under vacuum showed intense blue colour upon illumination by a hand-held UV lamp. The broad emission spectrum peaked at $448 \mathrm{~nm}$ (Figure S1). Remarkably, the fluorescence quantum yield was 4 to 6 times higher than in solutions. This effect can be attributed to the restriction of internal twisting motion resulting from adsorption of individual molecules on the solid support.

\subsection{Aggregation-Induced Emission Enhancement (AIEE)}

Increasing the proportion of water in a THF/water solution of $\mathbf{1}$ at constant dye concentration first led to a shift of emission from blue to turquoise, due to solvatochromic effect. Then, above $80^{\wedge} \%$ water in the medium, opacification of the medium occurred with emission of strong yellow light (Figure 4). Observation with the fluorescence microscope showed that the water/THF 95:5 mixture contained small fluorescent microcrystals, thus confirming the precipitation of the compound. Indeed, the procedure used is purely a reprecipitation method, commonly used for generating organic microcrystals in conditions very far from equilibrium. ${ }^{[44]}$ The fluorescence spectrum of the suspension 
had a maximum at $556 \mathrm{~nm}$ and, at this wavelength, the fluorescence intensity was multiplied by 19 in comparison with THF solution. According to the popular explanation, this clear aggregation-induced emission enhancement (AIEE) effect can be partially attributed to restriction of intramolecular motions, ${ }^{[27]}$ combined with the formation of excimers to account for the red shift of the emission spectrum with respect to dissolved molecules and molecules individually adsorbed on the Nylon filter. Alternatively, it has recently been shown that the electrostatic field induced on a single molecule by the crystalline environment is enough to rationalize AIE/AIEE effect. ${ }^{[45]}$ It is noteworthy that the strong red shift of the emission spectrum observed upon aggregation compares well with that reported for benzoxazole styryl dyes, the $\pi$-electron conjugated system of which is larger than that of compound $\mathbf{1}$. $^{[28,37,46]}$

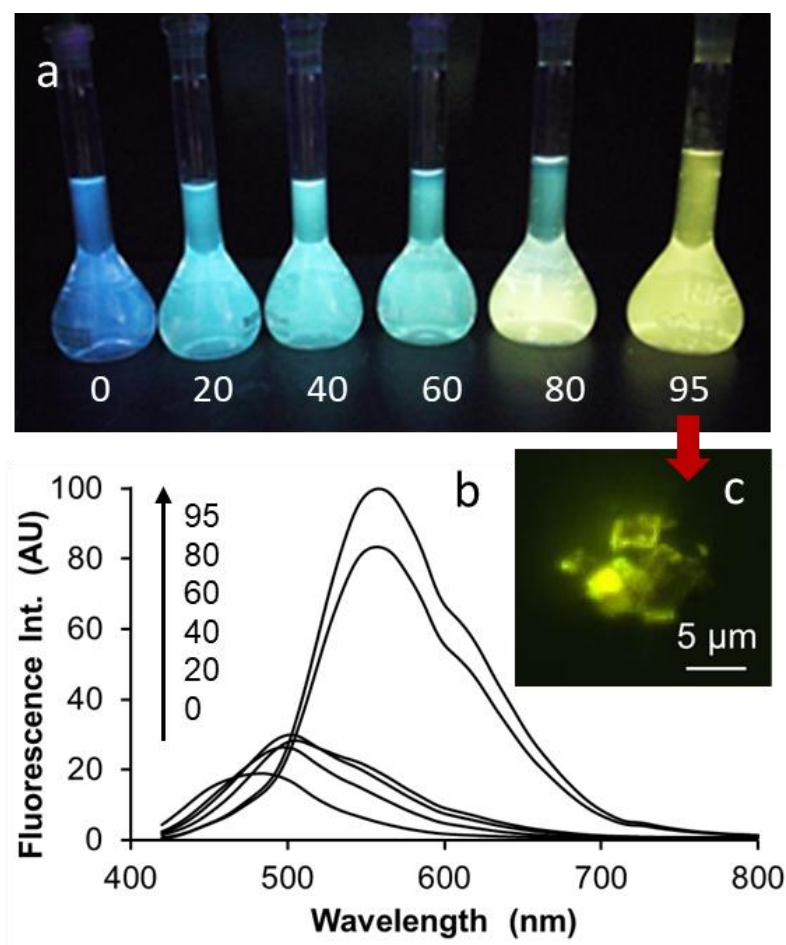

Figure 4. a) Picture of compound $1\left(2.6 \times 10^{-5} \mathrm{M}\right)$ in pure THF and various water/THF mixtures containing 20 , 40, 60, 80 and 95\% water upon illumination by a UV lamp at $365 \mathrm{~nm}$. b) Corresponding emission spectra with excitation at $380 \mathrm{~nm}$. Inset: Fluorescence microscopy image of 1 in water/THF 95:5 mixture $\left(\lambda_{\mathrm{ex}}=450-490 \mathrm{~nm}\right.$, $\lambda_{\mathrm{em}}>500 \mathrm{~nm}$ ).

\subsection{Mechanofluorochromism}

Solid-state spectroscopic data are collected in Table S2. Pristine compound $\mathbf{1}$ was a yellow powder, whose reflectance spectrum peaked at $425 \mathrm{~nm}$ and extended above $500 \mathrm{~nm}$ (Figure $5 \mathrm{a}$ and b). It was brightly emissive in the yellow-green under illumination by a UV lamp. The emission spectrum, recorded with an integrating sphere, peaked at $502 \mathrm{~nm}$. The solid-state photoluminescence quantum yield (PLQY) was 0.34, from 11 to 16 fold higher than in solutions. The fluorescence lifetime, measured with a fluorescence microscope, was found to be $4 \mathrm{~ns}$ in average (Figure S2).

Upon grinding with a mortar and a pestle, the colour of the powder changed very little to the naked eye, but the sample became brighter and clearly turned golden yellow under UV illumination. The maximum of the corresponding emission spectrum was slightly shifted to the red with respect to 
pristine powder while a shoulder appeared at $554 \mathrm{~nm}$. The PLQY increased to 0.39 . The average lifetime increased to $8 \mathrm{~ns}$ (Figure S3). No significant change was observed with time at room temperature.

To investigate thermal reversibility, the ground powder was successively heated for $48 \mathrm{~h}$ at 45 ${ }^{\circ} \mathrm{C}, 80^{\circ} \mathrm{C}, 125^{\circ} \mathrm{C}$ and $150{ }^{\circ} \mathrm{C}$. Measurements were made after each temperature step. A progressive blue shift of the emission maximum accompanied by a narrowing of the emission spectrum was observed with increasing temperature till $125{ }^{\circ} \mathrm{C}$. Meanwhile, the PLQY decreased linearly with increasing temperature (Figure 5c). The average lifetime of the sample heated at $125{ }^{\circ} \mathrm{C}$ was $6 \mathrm{~ns}$ (Figure S4). Heating to $150{ }^{\circ} \mathrm{C}$ induced no more changes in the spectral position and PLQY. Subsequent grinding and heating cycles were performed, leading to the expected changes in the position of the emission spectra. From the second cycle, the PLQY stabilized at around 0.29 for the ground powder, and around 0.24 for the sample heated at $125^{\wedge}{ }^{\circ} \mathrm{C}$ (Figure $5 \mathrm{~d}$ ).

Furthermore, the ground samples were exposed to solvent vapours for 4 days. With THF, only a very weak effect was detected on the emission spectrum, whereas with dichloromethane the emission spectrum began to resemble that of the pristine powder. Further exposure to dichloromethane vapours for up to 4 days generated a blue-shifted spectrum with low PLQY, very close to that obtained by heating at $125{ }^{\circ} \mathrm{C}$ (Figure S5). It is noteworthy that this exposure time is quite long, showing that our

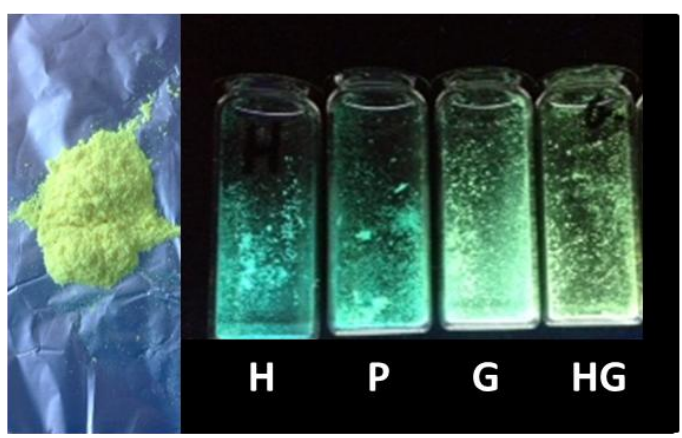

a
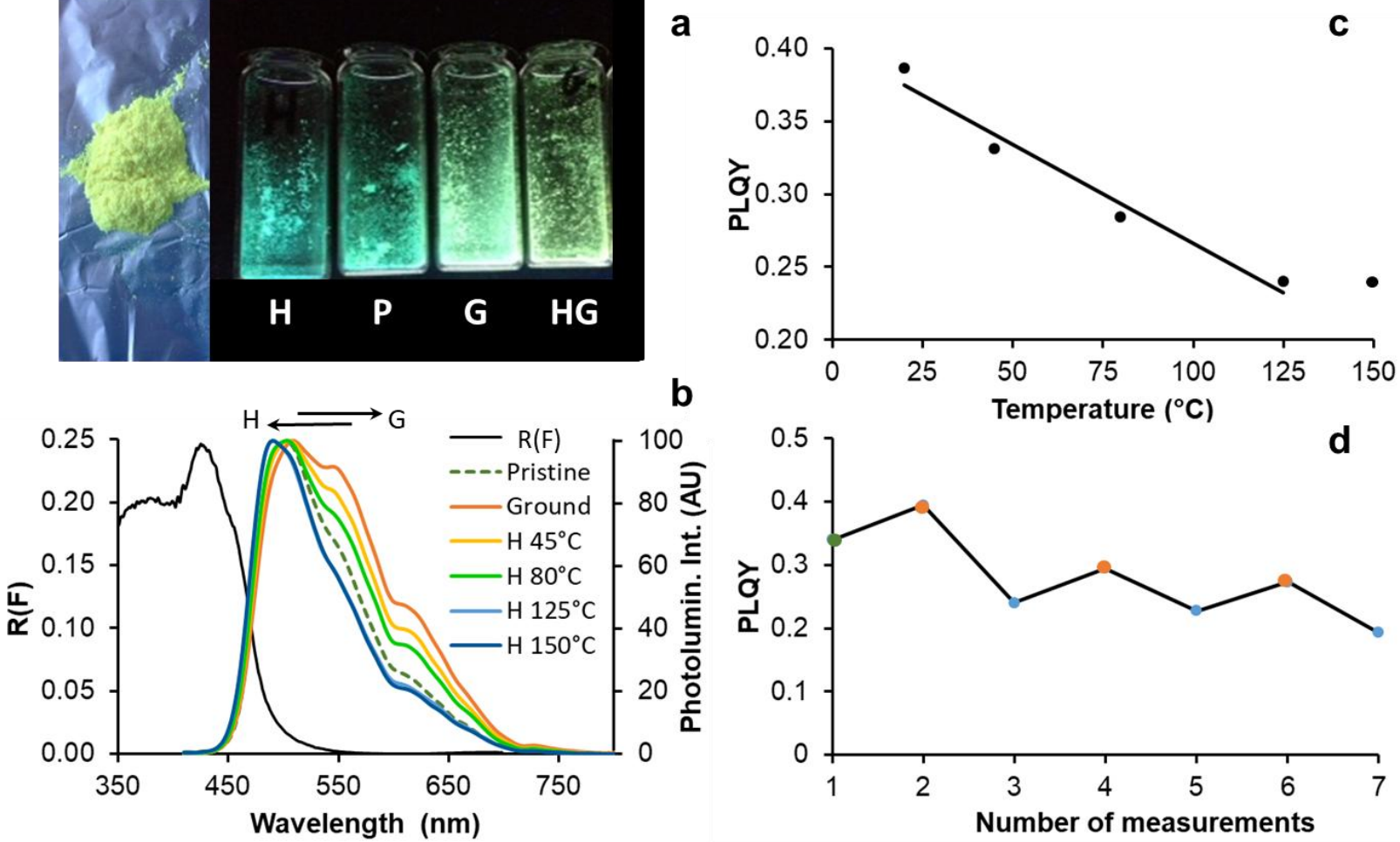

Figure 5. a) From left to right: Sample of pristine powder of 1 with day light. Samples of 1 examined upon illumination by a hand-hold UV lamp (365 nm): Ground, then heated at $150{ }^{\circ} \mathrm{C}(\mathrm{H})$; pristine $(\mathrm{P})$; ground $(\mathrm{G})$; ground, heated and then ground again $(\mathrm{HG})$. b) Reflectance of the pristine powder (left) and normalized photoluminescence spectra (right) of pristine powder (dashed line), ground powder (orange line), ground powder successively heated for $48 \mathrm{~h}$ at $45^{\circ} \mathrm{C}$ (yellow line), $80^{\circ} \mathrm{C}$ (green line), $125^{\circ} \mathrm{C}$ (blue line), $150^{\circ} \mathrm{C}$ (navy blue line). c) Evolution of the photoluminescence quantum yield (PLQY) depending on heating temperature from 20 to $150^{\circ} \mathrm{C}\left(\mathrm{R}^{2}=0.97\right)$. d) Evolution of photoluminescence quantum yield (PLQY) depending on many cycles of grinding (orange dots) and heating (blue dots) at $125^{\circ} \mathrm{C}$. 
compound is not very sensitive to the effect of solvent vapours. It is hard to explain why dichloromethane is more efficient, since compound $\mathbf{1}$ is well soluble in both solvents. A possibility is that dichloromethane induces greater mobility of the surface molecules compared with THF.

Additional experiments were then undertaken for the sake of comparison. First, some pristine powder was melted by heating with a gun in a test tube, and then rapidly frozen in liquid nitrogen. The resulting compact solid had an orange colour and emitted golden yellow light. The emission spectrum was strongly red-shifted $(550 \mathrm{~nm})$ with a rather low PLQY value of 0.20 (Figure 6).

Secondly, the big crystals grown from ethanol solution were observed. The light emitted by illumination with a hand-held UV lamp had a golden hue. The emission maximum was around $514 \mathrm{~nm}$ with a clear shoulder at $548 \mathrm{~nm}$, and the PLQY was around 0.12.

Finally, two powder samples were pressed using a small hydraulic press, under an operating pressure of $10 \mathrm{~T}$. Using pristine powder, the resulting pellet exhibited a broad emission spectrum, almost superimposable to that of a ground sample. Starting from ground powder, the pellet exhibited a markedly red-shifted spectrum with respect to the original sample (Figure 6).
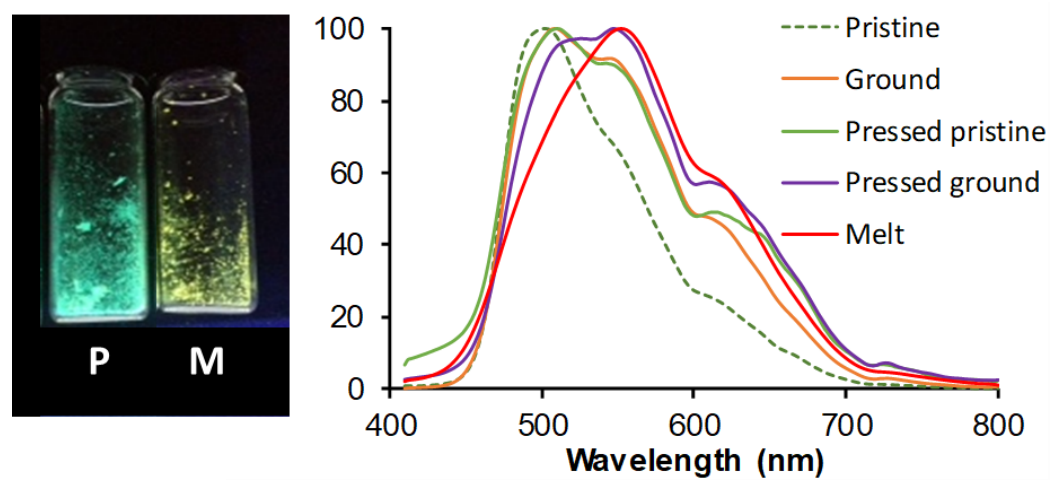

Figure 6. (a) Pristine compound $1(\mathrm{P})$ and melted-and-frozen sample (M) examined under a hand-hold UV lamp $(365 \mathrm{~nm})$. (b) From left to right: Normalized photoluminescence spectra of the pristine (dashed green line), ground (orange line), pressed pristine (solid green line), pressed ground (violet line) powders, and the meltedand-frozen sample (red line).

\subsection{X-Ray Diffraction and Microscopic Investigations}

To begin with, it must be emphasized that no degradation due to thermal or chemical treatment was detected in the processed samples, using thin layer chromatography. Observation of the samples with a polarization microscope gave information about their crystallinity (Figure 7, left column). After slow evaporation of an ethanol solution, compound $\mathbf{1}$ formed parallelepiped platelets, the largest side of which measured around $350 \mu \mathrm{m}$. The pristine powder directly issued from synthesis contained a large number of microcrystals, many of them with the same parallelepiped shape, but reduced dimensions. It is generally assumed that grinding leads to amorphization of the sample, while the thermal and solvent fuming treatments promote crystallization. Indeed, the ground powder came in the shape of lumps that did not allow polarized light to pass through, which is very typical of amorphous matter. After heating or solvent fuming, the powder showed very small bright dots, evoking tiny microcrystals. A new grinding process gave again the appearance of an amorphous compound. 


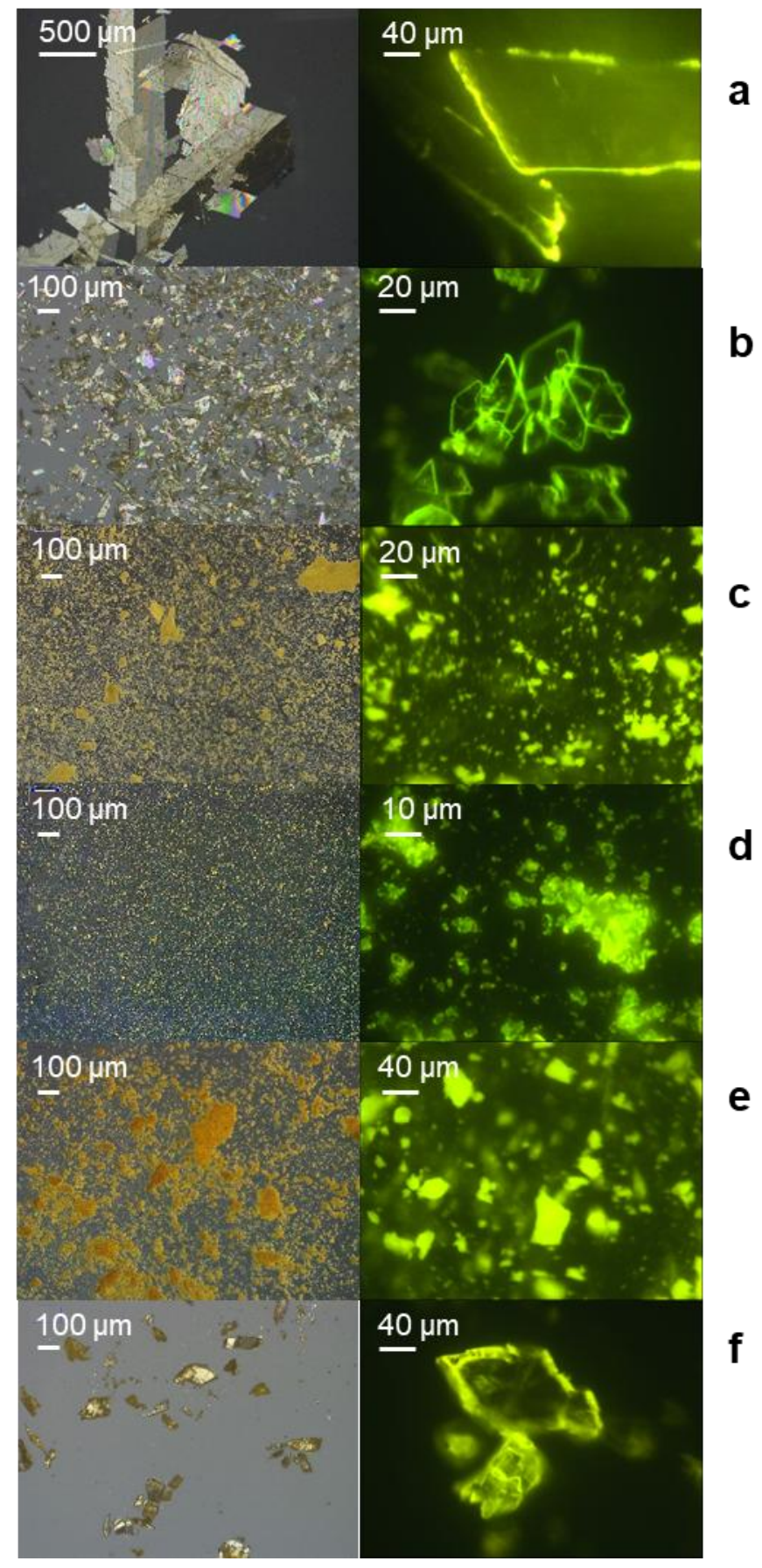

Figure 7. Optical polarization microscopy images (left column) and fluorescence microscopy images (right column, $\lambda_{\mathrm{ex}}=450-490 \mathrm{~nm}, \lambda_{\mathrm{em}}>500 \mathrm{~nm}$ ) of various samples of 1 . From top to bottom: a) crystals grown in ethanol; b) pristine powder; c) after grinding; d) after grinding and heating $\left(125^{\circ} \mathrm{C}\right)$; e) after grinding, heating and grinding again; f) melted and frozen. Fumed samples are not shown, but they were qualitatively comparable to heated samples.

The X-ray diffraction (XRD) spectra of powders confirmed these statements with some nuances. They were in perfect agreement with the spectrum calculated from X-ray data of a single crystal (Figure 8), indicating that the crystal packing mode was unchanged whatever the preparation conditions of the samples. The well-defined peaks obtained for the pristine, heated, and fumed powders support their good crystallinity. This was not the case for the ground powder, for which the 
broadened peaks reveal a very small crystal size. The presence of amorphous compound in this sample was not clearly detected by XRDP, contrary to observation by polarized microscopy. An explanation is that grinding during a short period of time only leads to a partially amorphous state, which indeed lacks long-range order, but without total disruption of the molecular order. ${ }^{[47]}$

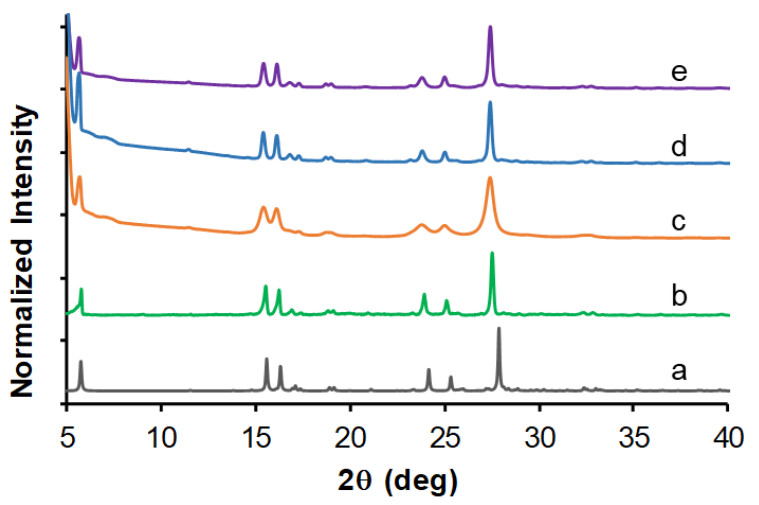

Figure 8. Powder X-ray diffraction (XRD) patterns (a) calculated from crystallographic data, and measured for (b) pristine microcrystalline powder, (c) ground powder, (d) powder ground then heated at $125^{\circ} \mathrm{C}$, (e) powder ground then fumed for $8 \mathrm{~h}$ with dichloromethane vapours.

When looking at the correlation with the spectroscopic characteristics, the green emission with maximum at 490-502 $\mathrm{nm}$ and short lifetime can be attributed to highly crystalline samples, while emission of golden light with a strong band at around $554 \mathrm{~nm}$ and long lifetime is associated to a compound with much lower crystallinity. From a molecular viewpoint, it is assumed that emission in organic crystals arises from single fluorophores influenced by their bulk surrounding environment, or from high-energy excimers (Figure 9) ${ }^{[8,45]}$ Whatever the precise nature of these emitting species, their energy levels can be modified by any change in the relative position of neighbouring molecules, such as those induced by amorphization of any degree, and they subsequently act as low-energy exciton traps, which in the present case would emit at longer wavelengths (Figure 9b). Because energy transfer processes are particularly active in the condensed state, a small number of traps are enough to explain the observed phenomenon. ${ }^{[8,48,49]}$ The reversible spectroscopic variations observed here can therefore be assigned to a mechanism close to mechanically induced phase transition. It can be thought that these variations are weak because most of the emitting species are relatively close to the original ones. Much more extensive grinding or other amorphization methods would be necessary to generate a real disorder at the molecular level.

This is the reason why the melted-and-frozen sample was initially prepared, because the quench cooling method is currently used to get a totally amorphous compound. ${ }^{[47]}$ Surprisingly, after division into small fragments, the melted-and-frozen sample appeared bright under the polarization microscope. A single fragment was mounted and examined by classical XRD technique. A pattern of reflections was obtained, but it was not possible to solve the crystal structure. This suggests that this sample was of polycrystalline nature. However, its emission spectrum was exactly what could have been expected for an amorphous sample. Besides, the emission of the large crystals grown in ethanol was red-shifted with respect to pristine powder, whereas crystallinity should be comparable in both samples. The same red shift of the emission spectrum was also observed after pressing microcrystals and ground powder. 
All these observations suggest that, independently from crystallinity, long-wavelength emission is related to other factors, like the sample heterogeneity and the length of the optical pathway through the sample.

Three mechanisms can be considered to take into account our whole set of observations. First of all, it can be imagined that low-energy exciton traps, emitting in the orange-yellow, are spontaneously formed at the interface of the many crystallites of various sizes and orientations that constitute the melted-and-frozen polycrystalline sample (Figure 9f). This is also the case for the agglomerates of microcrystals generated by adding a large proportion of water in THF, which present very close spectroscopic characteristics (Figure 4 and Table S1). Similarly, pressing induces close contact between differently oriented crystallites and molecules, and creates micro-regions rich in lowenergy exciton traps. Of course, pressing is a way to modify crystallinity and induce mechanochromism, but in the present case, the observed effect is most likely due to the compactness of the sample.

Secondly, the reddening of the emitted light with increasing the optical pathway could be explained by reabsorption of the short-wavelength component of the emitted light by molecules that absorb in the tail of the absorption spectrum, whereas the remaining orange component propagates through the solid until it is scattered by the surface. ${ }^{[50,51]}$ The significant overlap of the absorption and emission spectra suggests that this phenomenon may play here an essential role.

Finally, the study of the samples under the fluorescence microscope prompted us to consider an additional effect. In the large crystals grown from ethanol, as well as in the distinct platelets that constitute the pristine powder (Figure $7 \mathrm{a}$ and b, right column, and Figure S2), the large surfaces appeared dark while emission occurred solely at the edges. According to the literature, this selfwaveguided edge-emission is characteristic of a particular alignment of the transition dipoles in the crystal. ${ }^{[52-54]}$ Indeed, as seen above, the structuring effect of the cyano groups results in uniaxially oriented molecular packing, with molecules stacked nearly perpendicular to the diamond-shaped face of the crystal (Figure 9g). By analogy with closely-related PBO derivatives on which TD-DFT calculations have been performed, ${ }^{[46]}$ it can be assumed that the transition dipole moment is situated along the long axis of the molecule, and hence nearly vertical to the face of the crystal. Since emission occurs perpendicularly with respect to the transition dipole moment, the molecules tend to emit nearly parallel to the crystal ab plane. Due to the high refractive index of organic crystals, crystals of $\mathbf{1}$ thus act as waveguides, and emission is observed from the edges (Figure 9c). This peculiar optical property could have a magnifying effect on light reabsorption, and on the probability of encountering lowenergy exciton traps (the so-called surface defects), just by extending the path travelled by the emitted light in large crystals (Figure 9d). In contrast, this effect does not occur in the lumps of matter obtained by grinding, the whole surface of which emitted light homogeneously (Figure $7 \mathrm{c}$ and e, right column, Figures S3 and 9e). The thick pieces of the melted-and-frozen sample showed intermediate behaviour, because light was emitted from both the edges and numerous surface irregularities (Figures $7 \mathrm{f}$, right column, and 9f).

Finally, a link can be searched between the variations of the PLQY values and the position of the emission spectra. It is easy to understand that the melted-and-frozen sample, pressed pellets and macrocrystals grown in ethanol have a low PLQY value due to strong reabsorption. It can be assumed that the emitting species formed by grinding emit more efficiently than the pristine compound. But, this does not explain why the luminescence efficiency decreased between the first grinding/heating 
process and the following ones (Figure 5d), while thermal and chemical degradations of the samples can be discarded. An explanation could be that the PLQY value actually depends on many parameters linked to the size and shape of particles. Besides, it is known that many artefacts and difficulties arise from working with dyes in the condensed state. ${ }^{[51,55]}$ Sophisticated techniques combined with a theoretical approach would be necessary to rationalize these observations.

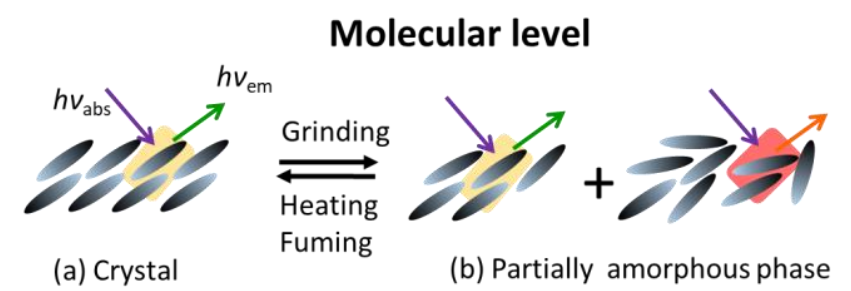

Macroscopic level

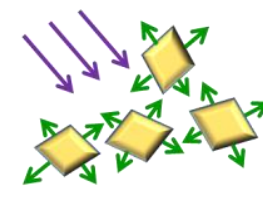

(c) Microcrystals

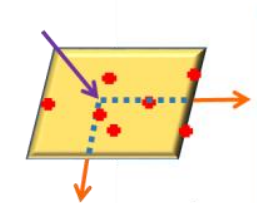

(d) Macrocrystal

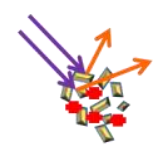

(e) Ground powder

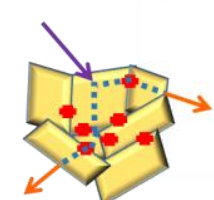

(f) Microcrystal agglomerates Polycrystalline solid

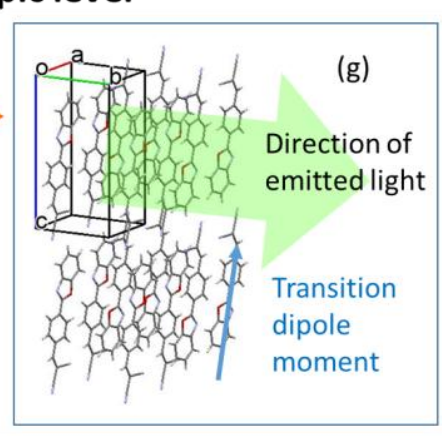

- $=$ Crystal defect, low-energy exciton trap

Figure 9. Schematic representation of fluorescence emission at the molecular scale in crystals (a) and in the partially amorphous phase (b), and at the macroscopic level in the various types of samples (c to f). g) Schematic illustration of the self-waveguided edge-emission mechanism showing the molecules stacked with their long axis almost perpendicular to the crystal face.

\section{Conclusions}

This work confirms our assumption that extending the electron conjugation of the PBO framework with a $\mathrm{CH}=\mathrm{CR}^{1} \mathrm{R}^{2}$ group inserted on the phenyl ring is enough to get AIEE behaviour, ${ }^{[28]}$ which is accompanied here by a MFC effect. The small dicyanovinyl group can be easily introduced in a large variety of dye molecules, and it would be instructive to know to what extent it can endow them with effective AIE/AIEE and MFC properties.

Regarding compound 1, its easy synthesis and the valuable fluorescence properties of the crystals obtained could lead to applications in the field of optical materials. This compound exhibits weak MFC behaviour compared to many compounds reported in the literature, which seem more suitable for applications as MFC materials. ${ }^{[56-66]}$ The reason is probably that in our case the crystal packing mode is not loose enough for a dramatic MFC effect. However, the weak MFC effect observed has allowed us to emphasize the importance of spectroscopic variations due to the size, heterogeneity and compactness of the samples. Confusion between these effects with the genuine MFC effect could explain the apparent lack of repeatability and reproducibility that may be 
encountered in this delicate field of research. It would now be instructive to study a compound that exhibits good MFC properties, and whose crystals show efficient self-waveguided edge-emission. The combination of these properties could be of special value for the development of strain sensors.

\section{Experimental Section}

Analytical grade absolute ethanol (Prolabo-VWR), tetrahydrofuran, dichloromethane, methanol and $n$ heptane from SDS were used as received. High pressure demineralized water (resistivity $18.2 \mathrm{M} \Omega . \mathrm{cm}$ ) was prepared with a Purelab flex apparatus from Elga Veolia. Malononitrile was purchased from Sigma Aldrich.

Synthesis. 2-(4'-Formylphenyl)benzoxazole $(100 \mathrm{mg}, 0.45 \mathrm{mmol})$, prepared as previously described ${ }^{[37]}$ and purified by chromatography, and malononitrile (30 $\mathrm{mg}, 0.45$ mmole) were solubilized in $30 \mathrm{~mL}$ ethanol. Copper $(5 \mathrm{mg}$ ) was added to the reaction medium that was then refluxed overnight. After cooling down to room temperature, the precipitated compound was collected by filtration and rinsed with $10 \mathrm{~mL}$ ethanol. Subsequent rinsing with dichloromethane afforded a yellow solution that was evaporated to dryness, giving $100 \mathrm{mg}$ of compound $\mathbf{1}$ as a yellow powder. Yield: $82 \%$.

2-(4'-(1,1-dicyanovinyl)phenyl)benzoxazole (1). $\mathrm{Mp}=229.3^{\circ} \mathrm{C} .{ }^{1} \mathrm{H} \mathrm{NMR}\left(300 \mathrm{MHz}, \mathrm{CDCl}_{3}\right) \delta$ (ppm): 8.43-8.38 (m, $\mathrm{H}_{9}$ and $\left.\mathrm{H}_{13}, 2 \mathrm{H}\right), 8.08-8.04\left(\mathrm{~m}, \mathrm{H}_{10}\right.$ and $\left.\mathrm{H}_{12}, 2 \mathrm{H}\right), 7.85-7.80\left(\mathrm{~m}, \mathrm{H}_{4}, 1 \mathrm{H}\right), 7.81$ (s, $\left.\mathrm{H}_{14}, 1 \mathrm{H}\right), 7.66-7.61\left(\mathrm{~m}, \mathrm{H}_{7}, 1 \mathrm{H}\right), 7.47-7.38\left(\mathrm{~m}, \mathrm{H}_{5}\right.$ and $\left.\mathrm{H}_{6}, 2 \mathrm{H}\right) .{ }^{13} \mathrm{C} \mathrm{NMR}\left(75 \mathrm{MHz}, \mathrm{CDCl}_{3}\right) \delta(\mathrm{ppm})$ : $161.0\left(\mathrm{Cq}, \mathrm{C}_{1}\right), 158.3\left(\mathrm{CH}, \mathrm{C}_{14}\right), 150.9\left(\mathrm{Cq}, \mathrm{C}_{2}\right), 142.0\left(\mathrm{Cq}, \mathrm{C}_{3}\right), 133.0\left(\mathrm{Cq}, \mathrm{C}_{8}\right), 132.2\left(\mathrm{Cq}, \mathrm{C}_{11}\right), 131.1$ $\left(\mathrm{CH}, \mathrm{C}_{10}\right.$ and $\left.\mathrm{C}_{12}\right), 128.4\left(\mathrm{CH}, \mathrm{C}_{9}\right.$ and $\left.\mathrm{C}_{13}\right), 126.3\left(\mathrm{CH}, \mathrm{C}_{5}\right), 125.2\left(\mathrm{CH}, \mathrm{C}_{6}\right), 120.6\left(\mathrm{CH}, \mathrm{C}_{4}\right), 113.4$ $\left(\mathrm{Cq}, \mathrm{C}_{16}\right), 112.3\left(\mathrm{Cq}, \mathrm{C}_{17}\right), 110.9\left(\mathrm{CH}, \mathrm{C}_{7}\right), 84.3\left(\mathrm{Cq}, \mathrm{C}_{15}\right)$. HRMS (DCI-CH$): m / z\left[\mathrm{M}+\mathrm{H}^{+}\right]$calcd. $\mathrm{C}_{17} \mathrm{H}_{10} \mathrm{~N}_{3} \mathrm{O}$ 272.0824; found: 272.0827. Anal. Calcd for $\mathrm{C}_{17} \mathrm{H}_{9} \mathrm{~N}_{3} \mathrm{O}: \mathrm{C}, 75.26 \% ; \mathrm{H}, 3.34 \%$; 15.49\%. Found: C, $74.87 \%$, H, 3.03\%; N, 15.24\%.

\subsection{Apparatus and methods}

NMR spectra, mass spectrum and microanalysis were recorded in the relevant services of Institut de Chimie de Toulouse (ICT). The ${ }^{1} \mathrm{H}$ NMR and ${ }^{13} \mathrm{C}$ NMR spectra were recorded on a Bruker AC300 spectrometer operating at $300 \mathrm{MHz}$ and $75 \mathrm{MHz}$, respectively. Protons and carbon atoms were numbered according to Figure 10. The mass spectrum was obtained with a Waters GCT Premier spectrometer using the chemical ionization technique with $\mathrm{CH}_{4}$. The microanalysis was obtained with an EA1112 elemental analyzer from CE Instruments. The melting point was measured on a Melting Point System MP50 apparatus. All microscopic and spectroscopic measurements were made at room temperature. The polarization stereomicroscope Leica S8 APO was equipped with a HD digital colour camera Leica MC170. The simple observation of the samples was made using a Zeiss Axioskop fluorescence microscope equipped with an Andor Luca camera. The excitation wavelength was 450$490 \mathrm{~nm}$, and the emission wavelength was set at above $500 \mathrm{~nm}$, using suitable filters. The intensityaveraged fluorescence lifetimes were measured on specks of the materials deposited on clean (First Contact, Photon Lines) cover slips, using a Nikon Ti-Eclipse inverted microscope equiped with a PicoQuant time correlated single photon counting system. Sub-nanosecond excitation at $405 \mathrm{~nm}$ was 
applied at $20 \mathrm{MHz}$ and emission was collected through a $405 \mathrm{~nm}$ notch filter (Semrock). The diffuse reflectance spectrum was obtained with an Agilent Technologies Cary series UV-Vis Spectrophotometer equipped with a $70 \mathrm{~mm}$ diameter-integrating sphere. The instrument was calibrated with a Spectralon reflectance standard (USRS-99-010). The familiar Kubelka-Munk transform was applied, so that the measured spectroscopic remission value was approximately proportional to the concentration of the absorbing species according to:

$$
F\left(R_{\infty}\right)=\left(1-R_{\infty}\right)^{2} / 2 R_{\infty}=K / S
$$

where $K$ is an absorption coefficient, $S$ is the anisotropic scattering coefficient and $R_{\infty}$ is the diffuse reflectance at "infinite thickness".

Both UV-visible absorption and fluorescence measurements in solution were performed with a Xenius SAFAS spectrofluorimeter using cells of $1 \mathrm{~cm}$ optical pathway. All fluorescence spectra were corrected. The fluorescence quantum yields $\left(\Phi_{\mathrm{F}}\right)$ of solutions were determined using the classical formula:

$$
\Phi_{\mathrm{Fx}}=\left(A_{\mathrm{s}} \times F_{\mathrm{x}} \times n_{\mathrm{x}}^{2} \times \Phi_{\mathrm{Fs}}\right) /\left(A_{\mathrm{x}} \times F_{\mathrm{s}} \times n_{\mathrm{s}}^{2}\right)
$$

where $A$ is the absorbance at the excitation wavelength, $F$ is the area under the fluorescence curve, and $n$ is the refraction index. Subscripts "s" and " $\mathrm{s}$ " refer to the standard and to the sample of unknown quantum yield, respectively. Quinine sulfate in $0.1 \mathrm{~N} \mathrm{HClO}_{4}\left(\Phi_{\mathrm{F}}=0.59\right)$ was taken as the standard. ${ }^{[6]}$ The absorbance of the solutions was equal or below 0.050 at the excitation wavelength. The error on $\Phi_{\mathrm{Fx}}$ values is estimated to be about $10 \%$. The photoluminescence of all solid samples was analyzed on the same apparatus using a $\mathrm{BaSO}_{4}$ integrating sphere. Solid samples were deposited on a metal support. The excitation source was scanned in order to evaluate the reflected light for the empty sphere $\left(L_{\mathrm{a}}\right)$, the samples facing the source light $\left(L_{\mathrm{c}}\right)$ and the sample out of the irradiation beam $\left(L_{\mathrm{b}}\right)$. The luminescence spectra were recorded with the sample facing the source light $\left(E_{\mathrm{c}}\right)$ and out from direct irradiation $\left(E_{\mathrm{b}}\right)$. The PM voltage was adapted to the measurement of reflected light and emission spectra, respectively, and proper correction was applied to take into account the voltage difference. The absolute photoluminescence quantum yield values $\left(\Phi_{\mathrm{PL}}\right)$ were calculated by a method based on the one developed by de Mello et al. ${ }^{[67]}$ using the formula:

$$
\Phi_{\mathrm{PL}}=\left[E_{\mathrm{c}}-(1-\alpha) E_{\mathrm{b}}\right] / L_{\mathrm{a}} \alpha
$$

with $\alpha=1-L_{\mathrm{c}} / L_{\mathrm{b}}$. The error on the $\Phi_{\mathrm{PL}}$ value was estimated to be about $20 \%$.

\subsection{Crystallography}

Crystal data were collected at low temperature (193(2)K) on a Bruker-AXS D8-Venture diffractometer equipped with a CMOS Area detector, with graphite-monochromated MoK $\alpha$ radiation $(\lambda=0.71073 \AA)$ by using phi- and omega scans. The structure was solved by using intrinsic phasing method (SHELXT) ${ }^{[68]}$ and all non-hydrogen atoms were refined anisotropically using the least-squares method on $\mathrm{F}^{2}{ }^{[69]}$ CCDC 1880966 contains the supplementary crystallographic data and can be 
obtained free of charge via www.ccdc.cam.ac.uk/conts/retrieving.html (or for the CCDC, 12 Union Road, Cambridge CB2 1EZ, UK; fax: +441223 336033; e-mail: deposit@ccdc.cam.ac.uk). Crystal Data for $1, \mathrm{M}=271.27$, triclinic, $P \overline{1}, \mathrm{a}=6.5485(2) \AA, \mathrm{b}=7.0431(2) \AA, \mathrm{c}=15.3745(5) \AA, \alpha=$ 84.437(1) ${ }^{\circ}, \beta=89.593(1)^{\circ}, \gamma=65.922(1)^{\circ}, V=643.94(3) \AA^{3}, Z=2$. Reflections collected: 26193 , independent reflections: $3186, \mathrm{R}_{\text {int }}=0.0641,191$ parameters, $\mathrm{R}_{1}[\mathrm{I}>2 \operatorname{sigma}(\mathrm{I})]=0.0567, \mathrm{wR}_{2}$ [all data] $=0.1518$. Largest diffusion peak and hole 0.254 and -0.277 e. $\AA^{-3}$.

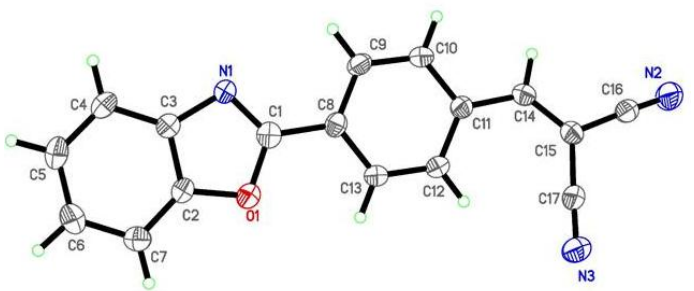

Figure 10. Molecular view of compound 1. Thermal ellipsoids are drawn at the $50 \%$ probability level.

The X-ray powder diffraction pattern of the pristine compound was recorded in the "Service Diffraction $X \mathrm{du}$ Laboratoire de Chimie de Coordination de Toulouse" with a $\theta-\theta$ XPert Pro Panalytical diffractometer. It was collected in transmission mode using a quartz capillary and the background was corrected. The XRD patterns of the ground, heated and fumed powders were recorded in the "Nano X platform of CEMES-CNRS". They were collected in reflexion mode using a BraggBrentano configuration on a Bruker D8 Advance diffractometer equipped with a 1D Lynxeye detector. The powders were placed in a sample holder with no background noise. In both cases, the diffractometers used a Copper anticathode $(\mathrm{Cu} \mathrm{K} \alpha 1=1.54059 \AA$ and $\mathrm{K} \alpha 2=1.54439 \AA)$.

\section{Acknowledgements}

ANR is gratefully acknowledged for funding (SUPERFON project \# ANR-17-CE07-0029-03). J. Wang thanks the Chinese Scholarship Council (CSC) for a Ph.D. funding. We are also indebted to Mr. Nicolas Ratel-Ramond (CEMES-CNRS) for for skillful measurements of XRPD patterns.

\section{Conflict of interest}

The authors declare no conflict of interest.

\section{References}

[1] R. Tan, S. Wang, H. Lan, S. Xiao S, Curr. Org. Chem. 2017, 21, 236-248.

[2] D. R. T. Roberts, S. J. Holder, J. Mater. Chem. 2011, 21, 8256-8268.

[3] F. Ciardelli, G. Ruggeri, A. Pucci, Chem. Soc. Rev. 2013, 42, 857-870.

[4] Y. Jiang, Mater. Sci. Eng. C 2014, 45, 682-689.

[5] P. Xue, J. Ding, P. Wang, R. Lu, J. Mater. Chem. C 2016, 4, 6688-6706. 
[6] C. Wang, Z. Li, Mater. Chem. Front. 2017, 1, 2174-2194.

[7] Z. Ma, Z. Wang, M. Teng, Z. Xu, X. Jia, ChemPhysChem 2015, 16, 1811-1828.

[8] L. Wilbraham, M. Louis, D. Alberga, A. Brosseau, R. Guillot, F. Ito, F. Labat, R. Métivier, C. Allain, I. Ciofini, Adv. Mater. 2018, 30, 1800817.

[9] S. P. Anthony, ChemPlusChem 2012, 77, 518-531.

[10] H. Ito, T. Saito, N. Oshi Wilbraham, N. Kitamura, S. Ishizaka, Y. Hinatsu, M. Wakeshima, M. Kato, K. Tsuge, M. Sawamura, J. Am. Chem. Soc. 2008, 130, 10044-10045.

[11] W. Z. Yuan, Y. Tan, Y. Gong, P. Lu, J. W. Y. Lam, X. Y. Shen, C. Feng, H. H. Y. Sung, Y. Lu, I. D. Williams, J. Z. Sun, Y. Zhang, B. Z. Tang, Adv. Mater. 2013, 25, 2837-2843.

[12] M. Liu, L. Zhai, J. Sun, P. Xue, P. Gong, Z. Zhang, J. Sun, R. Lu, Dyes Pigm. 2016, 128, 271-278.

[13] G. Zhang, J. Lu, M. Sabat, C. L. Fraser, J. Am. Chem. Soc. 2010, 132, 2160-2162.

[14] P. Xue, B. Yao, J. Sun, Q. Xu, P. Chen, Z. Zhang, R. Lu, J. Mater. Chem. C 2014, 2, 3942-3950.

[15] P. Xue, B. Yao, P. Wang, J. Sun, Z. Zhang, R. Lu, RSC Adv. 2014, 4, 58732-58739.

[16] P. Xue, P. Chen, J. Jia, Q. Xu, J. Sun, B. Yao, Z. Zhang, R. Lu, Chem. Commun. 2014, 50, $2569-2571$.

[17] Z. Zhang, P. Xue, P. Gong, G. Zhang, J. Peng, R. Lu, J. Mater. Chem. C 2014, 2, 9543-9551.

[18] S. Xue, X. Qiu, Q. Sun, W. Yang, J. Mater. Chem. C 2016, 4, 1568-1578.

[19] S. Ying, M. Chen, Z. Liu, M. Zheng, H. Zhang, S. Xue, W. Yang, J. Mater. Chem. C 2017, 5, 5994-5998. [20] S. J. Yoon, S. Y. Park, J. Mater. Chem. 2011, 21, 8338-8346.

[21] C. Botta, S. Benedini, L. Carlucci, A. Forni, D. Marinotto, A. Nitti, D. Pasini, S. Righettoce, E. Cariati, J. Mater. Chem. C 2016, 4, 2979-2989.

[22] Y. Jiang, D. Gindre, M. Allain, P. Liu, C. Cabanetos, J. Roncali, Adv. Mater. 2015, 27, 4285-4289.

[23] N. S. S. Kumar, S. Varghese, C. H. Suresh, N. P. Rath, S. Das, J. Phys. Chem. C 2009, 113, 11927-11935.

[24] Y. Ooyama, Y. Kagawa, H. Fukuoka, G. Ito, Y. Harima, Eur. J. Org. Chem. 2009, 5321-5326.

[25] J. Kunzelman, M. Kinami, B. R. Crenshaw, J. D. Protasiewicz, C. Weder, Adv. Mater. 2008, 20, $119-122$.

[26] Y. Sagara, T. Mutai, I. Yoshikawa, K. Araki, J. Am. Chem. Soc. 2007, 129, 1520-1521.

[27] Y. Q. Dong, J. W. Y. Lam, B. Z. Tang, J. Phys. Chem. Lett. 2015, 6, 3429-3436.

[28] C. Carayon, S. Fery-Forgues, Photochem. Photobiol. Sci. 2017, 16, 1020-1035 and references cited.

[29] A. Pucci, F. Di Cuia, F. Signori, G. Ruggeri, J. Mater. Chem. 2007, 17, 783-790.

[30] F. Cellini, S. Khapli, S. D. Peterson, M. Porfiri, Appl. Phys. Lett. 2014, 105, 061907.

[31] A. Battisti, P. Minei, A. Pucci, R. Bizzarri, Chem. Commun. 2017, 53, 248-251.

[32] Z. Zhang, Z. Wu, J. Sun, P. Xue, R. Lu, RSC Adv. 2016, 6, 43755-43766.

[33] J. Zhao, J. Peng, P. Chen, H. Wang, P. Xue, R. Lu, Dyes Pigm. 2018, 149, 276-283.

[34] A. Ghodbane, S. D’Altério, N. Saffon, N. D. McClenaghan, L. Scarpantonio, P. Jolinat, S. Fery-Forgues, Langmuir 2012, 28, 855-863.

[35] A. Ghodbane, N. Saffon, S. Blanc, S. Fery-Forgues, Dyes Pigm. 2015, 113, 219-226.

[36] A. Ghodbane, P. Bordat, N. Saffon, S. Blanc, S. Fery-Forgues, Dyes Pigm. 2016, 125, 282-291.

[37] A. Ghodbane, W. B. Fellows, J. R. Bright, D. Ghosh, N. Saffon, L. M. Tolbert, S. Fery-Forgues, K. M. Solntsev, J. Mater. Chem. C, 2016, 4, 2793-2801.

[38] Y. P. Tong, Acta Crystallogr. 2005, E61, o3076-o3078.

[39] A. Ghodbane, J. Colléaux, N. Saffon, R. Mahiou, J. P. Galaup, S. Fery-Forgues, ChemPlusChem 2013, 78, $185-191$.

[40] K. A. Willets, P. R. Callis, W. E. Moerner, J. Phys. Chem. B 2004, 108, 10465-10473. 
[41] T. Suhina, B. Weber, C. E. Carpentier, K. Lorincz, P. Schall, D. Bonn, A. M. Brouwer, Angew. Chem. Int. Ed. 2015, 54, 3688-3691

[42] T. Suhina, S. Amirjalayer, B. Mennucci, S. Woutersen, M. Hilbers, D. Bonn, A. M. Brouwer, J. Phys. Chem. Lett. 2016, 7, 4285-4290 and references cited.

[43] A-Y. Jee, E. Bae, M. Lee, J. Phys. Chem. B 2009, 113, 16508-16512.

[44] H. Oikawa, H. Nakanishi in Single Organic Nanoparticles (Eds.: H. Masuhara, H. Nakanishi, K. Sasaki) Springer, Berlin, 2003, pp. 17-30.

[45] D. Presti, L. Wilbraham, C. Targa, F. Labat, A. Pedone, M. C. Menziani, I. Ciofini, C. Adamo, J. Phys. Chem. C 2017, 121, 5747-5752.

[46] U. N. Yadav, H. S. Kumbhar, S. S. Deshpande, S. K. Sahoo, G. S. Shankarling, RSC Adv. 2015, 5, 4297142977.

[47] T. Einfalt, O. Planinšek, K. Hrovat, Acta Pharm. 2013, 63, 305-334.

[48] J. Su, T. Fukaminato, J. P. Placial, T. Onodera, R. Suzuki, H. Oikawa, A. Brosseau, F. Brisset, R. Pansu, K. Nakatani, R. Métivier, Angew. Chem. Int. Ed. 2016, 55, 3662-3666.

[49] I. Shulov, S. Oncul, A. Reisch, Y. Arntz, M. Collot, Y. Mely, A. S. Klymchenko, Nanoscale 2015, 7, 18198-18210.

[50] L. Bîrlă, F. Bertorelle, F. Rodrigues, S. Badré, R. Pansu, S. Fery-Forgues, Langmuir 2006, 22, 6256-6265.

[51] P.-A. Cazade, P. Bordat, S. Blanc, I. Baraille, R. Brown, Langmuir 2008, 24, 2252-2257.

[52] S. Mu, K. Oniwa, T. Jin, N. Asao, M. Yamashita, S. Takaishi, Org. Electron. 2016, 34, $23-27$.

[53] H.-H. Fang, J. Yang, J. Feng, T. Yamao, S. Hotta, H.-B. Sun, Laser Photonics Rev. 2014, 8, 687-715.

[54] S. Hotta, M. Goto, Adv. Mater. 2002, 14, 498-501.

[55] Y. Sagara, T. Kato, Angew. Chem. Int. Ed. 2011, 50, 9128-9132.

[56] H.-J. Kim, D. R. Whang, J. Gierschner, C. H. Lee, S. Y. Park, Angew. Chem. Int. Ed. 2015, 54, 4330-4333.

[57] Z. Ma, Y. Ji, Z. Wang, G. Kuang, X. Jia, J. Mater. Chem. C, 2016, 4, 10914-10918.

[58] S. Ito, G. Katada, T. Taguchi, I. Kawamura, T. Ubukata, M. Asami, CrystEngComm 2019, 21, 53-59.

[59] M. Tanioka, S. Kamino, A. Muranaka, Y. Ooyama, H. Ota, Y. Shirasaki, J. Horigome, M. Ueda, M. Uchiyama, D. Sawada, S. Enomoto, J. Am. Chem. Soc. 2015, 137, 6436-6439.

[60] T. Seki, N. Tokodai, S. Omagari, T. Nakanishi, Y. Hasegawa, T. Iwasa, T. Taketsugu, H. Ito, J. Am. Chem. Soc. 2017, 139, 6514-6517.

[61] Z. Ma, Z. Wang, X. Meng, Z. Ma, Z. Xu, Y. Ma, X. Jia, Angew. Chem. Int. Ed. 2016, 55, 519-522.

[62] Q. Liu, M. Xie, X. Chang, Q. Gao, Y. Chen, W. Lu, Chem. Commun. 2018, 54, 12844-12847.

[63] Y. Sagara, S. Yamane, M. Mitani, C. Weder, T. Kato, Adv. Mater. 2016, 28, 1073-1095.

[64] S.-J. Yoon, J. W. Chung, J. Gierschner, K. S. Kim, M.-G. Choi, D. Kim, S. Y. Park, J. Am. Chem. Soc. 2010, 132, 13675-13683.

[65] Y. Zhang, K. Wang, G. Zhuang, Z. Xie, C. Zhang, F. Cao, G. Pan, H. Chen, B. Zou, Y. Ma, Chem. Eur. J. 2015, 21, 2474-2479.

[66] C. Würth, M. Grabolle, J. Pauli, M. Spieles, U. Resch-Genger, Nat. Protoc. 2013, 8, 1535-1550.

[67] J. C. De Mello, H. F. Wittmann, R. H. Friend, Adv. Mater. 1997, 9, 230-232.

[68] G. M. Sheldrick, Acta Crystallogr. A 2015, 71, 3-8.

[69] G. M. Sheldrick, Acta Crystallogr. C 2015, 71, 3-8. 